\title{
Immunotherapy in head and neck squamous cell carcinoma: a narrative review
}

\author{
Shay Sharon ${ }^{1}$, R. Bryan Bell ${ }^{2}$ \\ ${ }^{1}$ Department of Oral and Maxillofacial Surgery, Hadassah Medical Center, Faculty of Dental Medicine, Hebrew University of Jerusalem, Jerusalem, \\ Israel; ${ }^{2}$ Earle A. Chiles Research Institute in the Robert W. Franz Cancer Center, Providence Cancer Institute, Portland, OR, USA \\ Contributions: (I) Conception and design: Both authors; (II) Administrative support: Both authors; (III) Provision of study materials or patients: None; \\ (IV) Collection and assembly of data: Both authors; (V) Data analysis and interpretation: Both authors; (VI) Manuscript writing: Both authors; (VII) \\ Final approval of manuscript: Both authors. \\ Correspondence to: R. Bryan Bell, MD, DDS, FACS, FRCS(Ed). Earle A. Chiles Research Institute in the Robert W. Franz Cancer Center, Providence \\ Cancer Institute, 4805 NE Glisan St. Suite 2N35, Portland, OR, USA. Email: Richard.bell@providence.org.
}

Background and Objective: In 2016, two anti-PD1 antibodies, nivolumab and pembrolizumab, were shown to improve overall survival in patients with recurrent/metastatic HNSCC and were approved by the US Food and Drug Administration (FDA) for use in the second line, cisplatin-resistant setting, although the overall response rates were only about $15 \%$. More recently, pembrolizumab was approved for use in the firstline $\mathrm{R} / \mathrm{M}$ setting as monotherapy in patients with CPS $>1$ or in combination with chemotherapy regardless of PD-L1 expression. Interestingly, while response rates with combination therapy were increased compared to pembrolizumab alone, the duration of response was shorter than might be expected. Based on a growing amount of evidence in other types of cancer treated with various combinations of immunotherapy, similar concepts are being studied in HNSCC, both in pre-clinical models and in clinical trials. Our objective is to provide a narrative review of the literature describing immunological concepts and novel approaches in the treatment of head and neck squamous cell carcinoma.

Methods: A review of the English literature published between January 1st, 1960 and January 2nd, 2021 was conducted using the Medline-PubMed and ClinicalTrials.gov databases. Main topics were selected for review, including basic immunology background, checkpoint inhibition, neoadjuvant immunotherapy, the combination of immunotherapy with radiation therapy and chemotherapy, intratumoral immunotherapy, and future prospects.

Key Content and Findings: This review presents recent advancements in treating HNSCC, focusing on the translation of immunological concepts into the standard of care. Key aspects of the immune response are delineated, and their implementation in various treatment modalities is then described. Cancer immunotherapy is a transformational approach for treating HNSCC, but overcoming existing cancer and its future spread poses a significant challenge. Despite the successful application of immunotherapy across multiple tumor types, durable response and cure remain elusive. Large-scale efforts are underway to test novel combinations of immunotherapy with other immunotherapy agents, targeted small molecules, chemotherapy, radiotherapy, and surgery. Future success will depend on better understanding of the synergies between therapies and their effect on the anti-cancer immune response. Providing the appropriate combination for each patient and unveiling the factors that determine a successful outcome is essential to the studies that should follow.

Conclusions: There is an ongoing effort, which is supported by an increasing body of evidence, to enhance response rates with combinations of immunotherapy with other immunotherapy agents, targeted small molecules, chemotherapy, radiation therapy, and surgery. The clinician and the scientist should be familiarized with basic immunologic concepts, key findings in recent clinical trials, and current indications for administering immunotherapy.

Keywords: Head and neck; cancer; immunotherapy 
Received: 07 April 2021; Accepted: 18 September 2021; Published: 10 September 2022.

doi: $10.21037 /$ fomm-21-48

View this article at: https://dx.doi.org/10.21037/fomm-21-48

\section{Introduction}

Head and neck squamous cell carcinoma (HNSCC) accounts for 600,000 new diagnosed cases worldwide each year (1), and approximately $60 \%$ of patients are expected to survive 5 years (2), a rate that has not changed significantly over the last decades. For many years, tobacco and alcohol consumption were regarded as the only known risk factors for the development of HNSCC, until more recently, when human papillomavirus (HPV) infection was also attributed as an independent risk factor for the development of oropharyngeal SCC. HPV-positive HNSCC is more prevalent among the young population (3), and its survival rate is $54 \%$ greater than its HPV-negative counterpart, which is generally caused by tobacco and alcohol consumption (4). The two conventional approaches to treat HNSCC are: (I) primary surgery followed by risk-adapted adjuvant radiation therapy (RT) or chemoradiotherapy (CRT); (II) definitive CRT. Treatment with either surgery and adjuvant therapy or definitive CRT achieves high rates of cure for HPV-positive HNSCC, while a high rate of therapeutic resistance characterizes HPV-negative tumors. Since HPV-positive patients are likely to live longer and experience the associated long-term toxicity of definitive CRT (5), there has been recent interest in radiation and/ or chemotherapy de-intensification. Both surgical and nonsurgical de-intensification strategies are actively pursued in numerous clinical trials (6-9). Retrospective studies of transoral surgery compared with definitive CRT suggest improved functional results in patients undergoing surgery with decreased gastrostomy tube dependency $(6,10,11)$ whereas a recent prospective study (ORATOR) reported contrasting results at an early time point (12).

On the other hand, poor outcomes and little change in overall survival (OS) over the last 50 years suggest that novel approaches are necessary for patients with HPV-negative disease. Regardless of etiology, recurrent or metastatic (R/ M) HNSCC poses an even greater challenge as only onethird of patients respond to treatment, and the median survival period is only 6-8 months (13). Immunotherapy has the potential to activate an immune response to target cancer cells by utilizing the function of the immune system to survey the body for abnormal cells and eliminate them continually. Numerous elements are engaged in the action of the immune system, but some of them have already been established as fundamental components of immunotherapy, such as programmed death receptor-1 (PD-1). Our review will describe recent advancements in treating HNSCC, focusing on the translation of immunological concepts into the standard of care. We will delineate key aspects of the immune response which are turning into "must-know" concepts in this world of personalized medicine. Next, we will review their implementation in various treatment modalities and provide a scientific background that will allow both the clinician and the scientist to comprehend their potential to be translated into the standard of care today and in the future. We present the following article in accordance with the Narrative Review reporting checklist (available at https://fomm.amegroups.com/article/ view/10.21037/fomm-21-48/rc).

\section{Methods}

The relevant English literature was identified by searching ClinicalTrials.gov and Medline via PubMed using the following search terms: Head and neck cancer, head and neck squamous cell carcinoma, immunotherapy, radiation in head and neck, radiotherapy, stereotactic radiation, intratumoral, intralesional, neoadjuvant, STING, microparticles, nanoparticles. To allow for a thorough review of immunology concepts, there was no limitation on the year of publication (Table 1). However, novel approaches were reviewed with an emphasis on publications from recent years. The data was reviewed using original research papers and clinical trials, while reviews were used mainly for a scientific background.

\section{Immunology}

\section{Immune response to cancer antigens}

Immune cells react to the tumor microenvironment (TME) by differentiating and varying their gene expression, hence constitute a means for understanding tumor behavior (14). The T-cell-mediated immune response is elicited by antigens presented by target cells or antigen-presenting cells (APC), such as dendritic cells (DC). Depending on their exposure to cytokines during DC activation, $\mathrm{CD}^{+}$ T-cells may differentiate into two major subpopulations, 
Table 1 The search strategy summary

\begin{tabular}{ll}
\hline Items & Specification \\
\hline Date of Search & January $2^{\text {nd }}, 2021$ \\
Databases and other sources searched & Medline via PubMed, ClinicalTrials.gov \\
Search terms used & Head and neck cancer, head and neck squamous cell carcinoma, immunotherapy, \\
& $\begin{array}{l}\text { radiation in head and neck, radiotherapy, stereotactic radiation, intratumoral, } \\
\text { intralesional, neoadjuvant, STING, microparticles, nanoparticles. }\end{array}$ \\
Timeframe & January $1^{\text {st }}, 1960-$ January $2^{\text {nd }}, 2021$ \\
Inclusion and exclusion criteria & $\begin{array}{l}\text { Included: Original research, reviews, clinical trials. Excluded: Languages other than } \\
\text { English }\end{array}$ \\
Selection process & $\begin{array}{l}\text { The selection was conducted independently by SS and RBB, followed by joint } \\
\text { deliberation and shared decision making }\end{array}$ \\
\hline
\end{tabular}

T helper 1 (Th1) and T helper 2 (Th2). Broadly, Th1 differentiation drives cellular immunity against viruses and infections, while Th2 differentiation directs the immune response to control parasitic diseases. Production of IL-12 and interferon-gamma (IFNg) by macrophages and natural killer cells, respectively, cause T-cells to differentiate into Th1 cells, while IL-4 may trigger Th2 cell differentiation. When stimulated in the presence of activated $\mathrm{CD}^{+} \mathrm{T}$-cells, $\mathrm{CD}^{+}{ }^{+} \mathrm{T}$-cells expand into effector T-cells that can attack target cells. Following initial expansion, $\mathrm{CD}^{+}$cells may also differentiate into regulatory T-cells (Treg's), which can suppress $\mathrm{CD}^{+}$cells and anti-tumor immunity.

Treg's are a subset of $\mathrm{CD}^{+}{ }^{+} \mathrm{T}$-cells that possess a central role in adjusting the immune response and preventing autoimmunity. They secrete IL-10 and transforming growth factor beta (TGFb), which inhibit immune response; they consume IL-2 that is required to trigger the proliferation of antigen-activated T-cells; and they express FOXP3, which in turn inhibits the expression of IL-2 and stimulates the expression of Cytotoxic T-lymphocyte-associated protein 4 (CTLA-4) and CD25. Expression of CD25 causes consumption of IL-2, which is a vital component of the immune response (15). The amount of Treg's within a tumor and in the peripheral blood of cancer patients is high, and they are presumed to suppress the immune response against cancer (16).

Since the anti-cancer response is directed towards intracellular mutated peptides, Th1 type responses are optimal to help drive $\mathrm{CD}^{+}$mediated cellular immunity against the tumor. The differentiation of $\mathrm{CD}^{+}$cells into Th1 cells promotes $\mathrm{CD}^{+} \mathrm{T}$-cell-mediated adaptive immunity (17), and it has been associated with anti-cancer response and better clinical outcomes (18). Conversely, serum analysis of HNSCC patients demonstrated elevated levels of Treg's (19) and Th2 cytokines, such as IL-4 and IL-10, compared to healthy individuals (20). Th2-associated cytokines have also been linked to a worse clinical outcome in cancer patients (21).

Mature DC are believed to play a central role by presenting tumor-specific antigens in the lymph nodes, thus priming and activating tumor-specific T-cells $(22,23)$. On the other hand, immature DC are characteristic of a steady-state condition, in which low expression of major histocompatibility complex (MHC) and co-stimulatory molecules elicits immune tolerance towards the antigens that they present $(23,24)$. Different subsets of DC populations possess variable capabilities for stimulating cytotoxic T-cells; some exist within the tumor, and their amount has been shown to predict tumor regression and clinical outcome (25).

Thus, as the HNSCC tumor progresses, the immune response shifts to suboptimal Th2 phenotypes and suppressive Treg populations (21), $\mathrm{CD} 8^{+}$control of cancer cells becomes limited, and DC drive towards tolerance rather than prime new $\mathrm{CD} 8^{+}$responses.

Since surgery plays a vital role in the treatment of most solid tumors, including HNSCC, its immunological effect on cancer cells is under investigation. Krall et al. demonstrated in the murine model that the systemic inflammatory response following surgery leads to reactivation of tumors, which correlated to earlier reports of the outgrowth of distant metastases in post-mastectomy cancer patients that underwent late breast reconstruction $(26,27)$. The notion that the surgery itself is pro-metastatic suggests that perioperative approaches to overcoming postoperative immunosuppression may be warranted. 


\section{Checkpoint inbibition}

CTLA-4, PD-1, and programmed death-ligand 1 (PD-L1) are crucial components of the immune response being targeted by immunotherapy. CTLA-4 interferes with the interaction between $\mathrm{APC}, \mathrm{CD}^{+}$, and $\mathrm{CD}^{+}$; blocking CTLA-4 allows this interaction to reform, and it also causes depletion of Treg's. PD-1 is a receptor expressed on activated T-cells, B-cells, and myeloid cells; it acts to downregulate immune response, and its ligands, PD-L1 and PD-L2, are expressed on both normal and cancerous cells (28). PD-L1 serves as a regulator of the inter-relationship between the tumor and the immune response and, as such, is suggested to play a prognostic role in the survival of HNSCC patients (29). PD-L1 is expressed in 50-60\% of HNSCC and at a higher level in intra-tumoral Treg cells than peripheral Treg cells (30). Using the combined positive score (CPS), which takes both the tumor cells and the surrounding immune cells into account, the expression level reaches $85 \%$ (31,32). Immunotherapeutic drugs such as nivolumab and pembrolizumab act by blocking PD-1 and lead to improved survival in many solid tumor types, including HNSCC.

\section{Immunotherapy}

\section{Immunotherapy in HNSCC}

In 2016, the FDA approved nivolumab and pembrolizumab as a second-line treatment for R/M HNSCC, and in 2019 pembrolizumab, either alone or in combination with chemotherapy, was approved as a first-line treatment, establishing a new standard of care. Prior to that, the only monoclonal antibody being used in HNSCC was the epidermal growth factor receptor (EGFR) inhibitor, cetuximab. It was approved by the FDA in 2006 for treating HNSCC in the definitive setting, and together with concurrent RT, it was considered a standard of care systemic option $(33,34)$. Recently, however, the results of two large phase 3 trials, including RTOG 1016 and the De-ESCALaTE trial comparing cetuximab to cisplatin in the definitive treatment of patients with $\mathrm{p} 16^{+} \mathrm{HPV}$-related oropharynx cancer were reported: In both RTOG 1016 (a randomized, multi-center, non-inferiority trial; $\mathrm{n}=805$ ) (35) and De-ESCALaTE (open-label, randomized controlled phase 3 trial, $n=334$ ) (36), cetuximab was inferior to cisplatin for OS and was more toxic.

In contrast, more promising results were reported in clinical trials studying the safety and efficacy of nivolumab and pembrolizumab, including overall response rate (ORR), OS, progression-free survival (PFS), and adverse events. Among these studies, CHECKMATE-141 was a phase 3 randomized trial conducted on 361 heavily pretreated R/M HNSCC patients that showed longer OS in the nivolumab group (7.5 months, 95\% CI: 5.5-9.1) than standard chemotherapy regimens (5.1 months, $95 \% \mathrm{CI}$ : 4.0-6.0), with OS hazard ratio for death 0.70 (37). This trend was also apparent after 24 months of follow-up, exhibiting OS of $16.9 \%$ (95\% CI: $12.4-22.0 \%$ ) vs. $6.0 \%$ (95\% CI: $2.7-11.3 \%$ ), and median survival of 7.7 (95\% CI: 5.7-8.8) months vs. 5.1 (95\% CI: 4.0-6.2) months, nivolumab $v s$. investigator's choice, respectively (38). These results were confirmed at ASCO 2019, showing similar OS rates among patients older or younger than 70 years (39). Pembrolizumab and investigator's choice were compared in KEYNOTE-040 (a randomized, open-label, phase 3 trial, $n=495$ ) and, while pembrolizumab was found to be clinically beneficial, the survival endpoints in the study were not met 8.4 (95\% CI: 6.4-9.4) months vs. 6.9 (95\% CI: 5.98.0) months, presumably because of cross-over in the study group (40). However, a phase 3 clinical trial in the first-line R/M setting, KEYNOTE-048 (a randomized, open-label, phase 3 trial, $n=882$ ), recently showed that pembrolizumab alone improved survival compared to cetuximab with chemotherapy in PD-1 positive HNSCC patients (CPS $>20,14.9$ vs. 10.7 months, HR 0.61, 95\% CI: 0.45-0.83, $\mathrm{P}=0.0007$; CPS >1, 12.3 vs. 10.3 months, HR $0.78,95 \%$ CI: 0.64-0.96, $\mathrm{P}=0.0086$ ), and pembrolizumab with chemotherapy improved survival compared to cetuximab with chemotherapy regardless of PD-1 status (13.0 vs. 10.7 months, HR $0.77,95 \%$ CI: $0.63-0.93, \mathrm{P}=0.0034)$ (41).

Adverse events following treatment of HNSCC with pembrolizumab appeared in $62-64 \%$ of patients and consisted of fatigue, decreased appetite, nausea, pruritus, rash, and hypothyroidism. Grade 3-4 adverse events included hyponatremia, elevated alanine aminotransferase and aspartate aminotransferase, atrial fibrillation, congestive heart failure, and pneumonitis, which had also led to the death of a single patient during the KEYNOTE-055 trial (42-44). Grade 3 and above adverse events were evident in $13 \%$ of patients treated with pembrolizumab vs. $36 \%$ among patients administered with investigator's choice in KEYNOTE-040 trial (40), and similar values were also reported in CHECKMATE-141 trial (15.3\% vs. 36.9\%, nivolumab and investigator's choice, respectively) (38). The relatively low rate of adverse events underlines the potential of immunotherapy as an alternative to the 
Table 2 Selected combination immunotherapy clinical trials in head and neck cancer

\begin{tabular}{|c|c|c|c|c|}
\hline Study & NCT ID & Phase & Drugs and treatment & HNSCC population \\
\hline CHECKMATE-714 & NCT02823574 & 2 & Nivolumab + ipilimumab vs. nivolumab + placebo & $\mathrm{R} / \mathrm{M}$ \\
\hline IMSTAR-HN & NCT03700905 & 3 & $\begin{array}{l}\text { Surgical resection + neoadjuvant/adjuvant nivolumab and } \\
\text { ipilimumab followed by adjuvant CRT vs. surgical resection } \\
\text { followed by adjuvant standard-of-care CRT }\end{array}$ & Primary \\
\hline CONDOR (c) & NCT02319044 & 2 & \multirow{2}{*}{$\begin{array}{l}\text { MEDI4736 (anti-PD-L1) +/- tremelimumab (anti-CTLA-4) vs. } \\
\text { standard-of-care chemothrapy }\end{array}$} & \multirow{2}{*}{$\mathrm{R} / \mathrm{M}$} \\
\hline EAGLE (c) & NCT02369874 & 3 & & \\
\hline INDUCE-3 & NCT04128696 & $2 / 3$ & $\begin{array}{l}\text { Pembrolizumab + feladilimab (ICOS inhibitor) vs. } \\
\text { pembrolizumab + placebo }\end{array}$ & $\mathrm{R} / \mathrm{M}$ \\
\hline KEYSTROKE & NCT03546582 & 2 & Pembrolizumab + SBRT & $\mathrm{R} / \mathrm{M}$ or $2^{\text {nd }}$ primary \\
\hline \multirow[t]{3}{*}{ Keynote-717 } & NCT03386357 & 2 & Pembrolizumab + RT vs. pembrolizumab alone & $\mathrm{R} / \mathrm{M}$ \\
\hline & NCT03521570 & 2 & Nivolumab + RT & $\mathrm{R} / \mathrm{M}$ or $2^{\text {nd }}$ primary \\
\hline & NCT02289209 & 2 & Pembrolizumab + re-irradiation & Inoperable or $2^{\text {nd }}$ primary \\
\hline
\end{tabular}

HNSCC, head and neck squamous cell carcinoma; PD-L1, programmed death-ligand 1; CTLA-4, cytotoxic T-lymphocyte-associated protein 4; RT, radiation therapy; SBRT, stereotactic body radiation therapy; CRT, chemoradiotherapy; R/M, recurrent or metastatic.

current standard of care treatment, which is often not well tolerated. This benefit was especially evident in the final analysis of KEYNOTE-048 trial, which demonstrated a significantly lower rate of grade three and above adverse events among the pembrolizumab alone group (55\%) than in the chemotherapy combination groups $(83 \%$ for chemotherapy and pembrolizumab, $85 \%$ for chemotherapy and cetuximab) (41). Incorporating immunotherapy into the treatment regimen of R/M HNSCC patients can also address the unmet need for improving quality of life (QOL). Analysis of questionnaires given to patients enrolled in KEYNOTE-040 demonstrated a stable QOL in pembrolizumab-treated patients, contrasting a decline in QOL among patients treated with standard of care (methotrexate, docetaxel, or cetuximab) (45).

In recent years, the number of immunotherapy trials for HNSCC has increased substantially, and since immunotherapy as a single modality treatment has yielded low response rates in the $\mathrm{R} / \mathrm{M}$ setting, trials are concentrating on the combination of immunotherapeutic agents, concurrent immunotherapy and chemotherapy, and concurrent immunotherapy and RT. Table 2 summarizes selected combination immunotherapy clinical trials in HNSCC.

\section{Neoadjuvant immunotherapy}

The propagation of immunotherapy into the neoadjuvant realm can prime both local and systemic immunity by targeting cancer cells and counteract immunosuppression at an early stage, tackling the potential suppression imposed by the surgery itself. It has also facilitated the study of resected specimens to evaluate responders $v s$. nonresponders on a cellular level $(46,47)$ and to examine the response to treatment and adjust it accordingly. While data regarding a possible link between pathological features and regression of HNSCC is still limited, data from other solid tumors demonstrated a correlation between several pathologic features and tumor regression: In a study of twenty cases of non-small-cell lung carcinoma (NSCLC) that received neoadjuvant nivolumab followed by surgical resection, tumor's regression was correlated with a dense population of tumor-infiltrating lymphocytes (TIL) and macrophages; tertiary lymphoid structures; cholesterol 
clefts; neovascularization and proliferative fibrosis (48). Accumulation of TIL was also associated with pathologic response in high-risk resectable stage 3-4 melanoma patients treated with a single dose of anti-PD-1, which likewise was associated with a clinical benefit (47). These data emphasize the potential of neoadjuvant therapy, not only for better loco-regional and systemic control of the disease but also as a method for further understanding the underlining pathology and for tailoring the treatment to the individual.

Pembrolizumab has been tested in the neoadjuvant setting prior to surgery in HPV-negative HNSCC (NCT02296684), with results recently published (49). In this trial, pathologic tumor response (pTR) was quantified as the proportion of the resection bed with tumor necrosis, keratinous debris, and giant cells/histiocytes: pTR-0 $(<10 \%)$, pTR-1 (10-49\%), and pTR-2 ( $\geq 50 \%)$ in 36 patients. While there were no complete pathologic responses (pCR) observed after neoadjuvant pembrolizumab, pTR-2 occurred in the surgical specimens of 8 patients (22\%), and pTR-1 occurred in 8 additional patients (22\%). Overall, pTR of $>10 \%$ was observed in 16 of 36 patients (44\%). Down-staging of cancer (defined as pathologic stage lower than clinical stage) after neoadjuvant pembrolizumab occurred in 7 patients (19\%), but only one patient had what would be considered a major pathologic response (MPR). These data provided the rationale for KN689, the ongoing phase 3 registrational trial sponsored by Merck (NCT03765918). Pathological response was also reported in a phase 2 clinical trial that included 29 oral cavity SCC patients treated with neoadjuvant nivolumab $v s$. a combination of nivolumab and ipilimumab. Clinical staging at diagnosis was T2 for twenty patients and T3-4 for the remaining nine. Pathologic response was as high as $54 \%$ and $73 \%$, pathologic downstaging of $53 \%$ and $69 \%$, RECIST response of $13 \%$ and $38 \%$, and 1 -year PFS of $85 \%$ and $89 \%$ (95\% CI: 72.4-99.7\%; 78.3-100\%), nivolumab vs. nivolumab+ipilimumab, respectively. Four patients demonstrated major or complete response, and the individual description of these patients is of particular interest: Following one cycle of neoadjuvant nivolumab, a cT4aN2b patient was treated with definitive CRT due to the extent of the disease, which resulted in complete metabolic response and remaining disease-free at 34 months. Another patient demonstrated $70 \%$ pathologic response at the primary tumor site but developed distant metastases, which were treated with adjuvant nivolumab and resulted in a complete metabolic response (50). Although the amount of patients in these trials is relatively small, and complete response is evident only in a limited set of patients, the potential of neoadjuvant immunotherapy to improve survival is substantial. Moreover, it allows for the study of response while administering treatment and adjusting it accordingly. Numerous trials are ongoing (Table 3).

\section{The immunogenic radiation}

$\mathrm{RT}$ is an essential component of treating many types of cancer, and for many years, it was generally regarded as immunosuppressive, mostly due to its cytotoxic effect on leukocytes, leading to lymphopenia and impaired leukocyte function $(51,52)$. However, more recent studies demonstrated a synergy between RT and the immune system that under certain circumstances may induce the immune response (53). This synergy can be subclassified into two types: In the first type, RT functions as an in situ vaccine that has the potential to boost immune control of distant disease $(30,54)$. In the second type of synergy, RT promotes a distinctive mechanism of immunogenic cell death, characterized by the release of activating signals that initiate the attraction of DC into the tumor and phagocytosis of irradiated tumor cells $(52,55,56)$. Induction of vascular density and leakiness by the tumor is counteracted by combined RT and immunotherapy, leading to more significant infiltration of $\mathrm{CD} 8^{+} \mathrm{T}$-cells and induction of migration and extravasation of leukocytes into the tumor $(52,57)$. Importantly, RT alone may lead to immunosuppression by the accumulation of Treg cells inside the tumor due to their radio-resistance; however, this process can be inhibited by administering concurrent immunotherapy $(52,58)$. These data demonstrate the potential of combining RT and immunotherapy to improve response rates and has led to a growing interest in this combination (59).

\section{SBRT and bypo-fractionated radiation therapy}

The conventional RT that most patients undergo is protracted fractionation RT, during which small doses of radiation (around 2 Gy per fraction) are delivered daily. This method presumably allows for normal tissue to undergo repair better than tumor tissue (60), therefore targeting the radiation effect at cancer cells more than at their surrounding healthy counterparts. A different approach for administering RT is stereotactic body radiation therapy (SBRT) which is, in essence, a precise delivery of high dose hypo-fractionated radiation to a target of either a single 
Table 3 Selected neoadjuvant immunotherapy trials in head and neck cancer

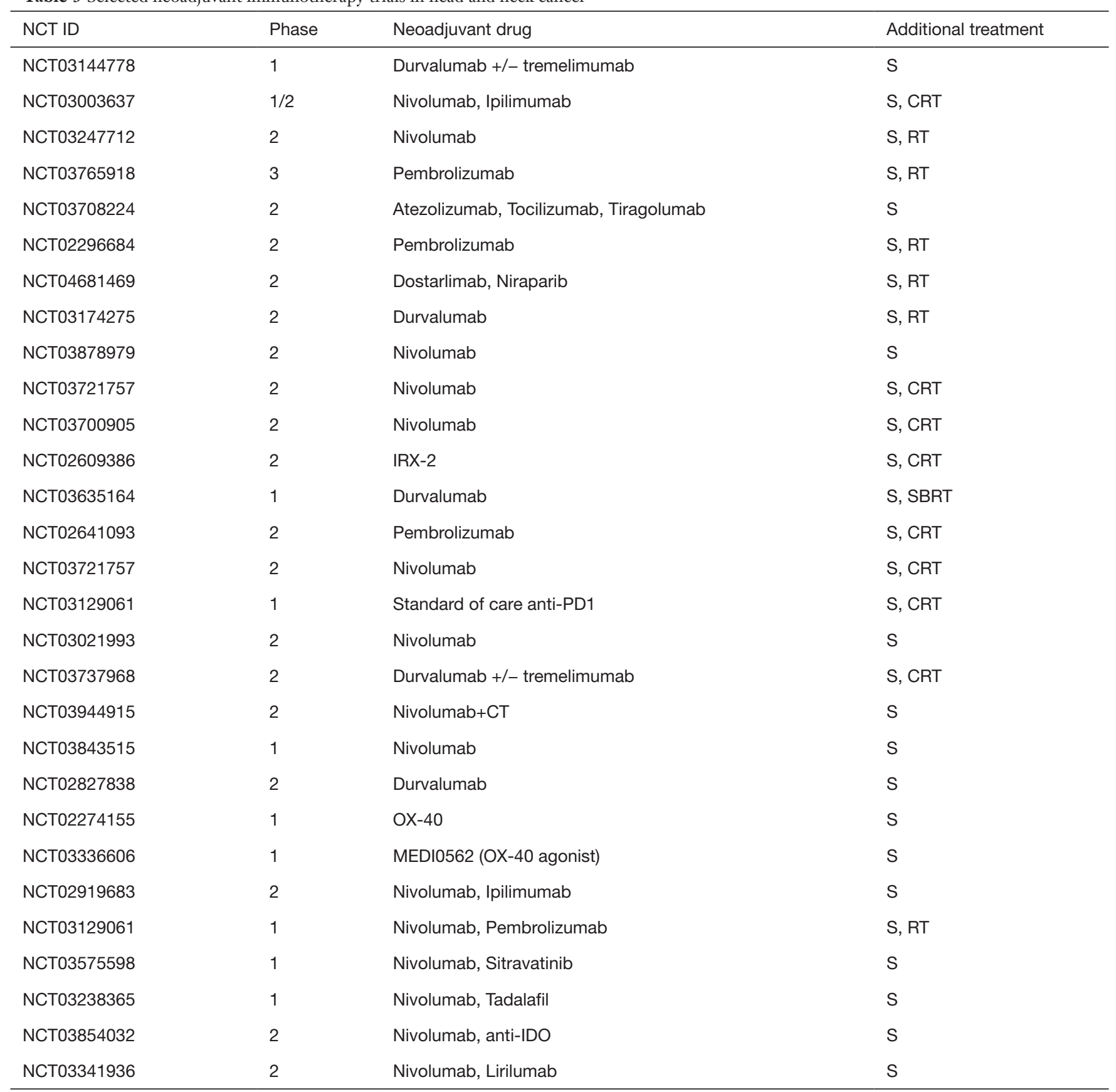

$\mathrm{S}$, surgery; CRT, chemoradiotherapy; RT, radiation therapy; CT, chemotherapy; SBRT, stereotactic body radiation therapy.

dose or a limited number of doses. This method enables a high dose of radiation to be focused on a specific location while maintaining a steep dose gradient beyond (61). For a group of medically unfit patients, administering primary hypo-fractionated RT has already proved to be a viable treatment alternative, minimizing the related toxicities and yielding impressive local control (LC) and OS rates $(\mathrm{n}=24$ lesions in 21 patients, $25 \%$ complete response, $67 \%$ partial response) $(62)(\mathrm{n}=55$ lesions in 44 patients, tumor control at one year $83.3 \%$ and $60.6 \%$, primary and recurrent groups, respectively) (63). SBRT is also being employed to treat oligometastatic disease, a state defined 
in 1995 by Hellman and Weichselbaum as an intermediate condition on a spectrum extending from localized disease to a rapidly advancing systemic disease. According to this notion, a disease that has spread to the lymph nodes is considered aggressive, and the involved nodes also serve as a nexus for seeding cancer (64). On the other hand, the oligometastatic state might signify an advanced disease that has not yet evolved into a systemic state; hence, it can be potentially cured. Various attempts are being made to treat oligometastatic disease, including surgical resection of metastases (metastasectomy) and SBRT. Interestingly, metastasectomy was proven beneficial for lung metastases, prolonging life, and even potentially curative in a selected group of patients (65). Metastases are also managed nonsurgically, utilizing SBRT to target a specific focus while minimizing the undesirable peripheral effect. Although SBRT can be seen, like surgery, as an opportunity for annulling a cancer site, the effect of SBRT on metastases is also hypothesized to be inherently immunogenic by turning the irradiated site into a vaccine that primes the immune response both locally and systemically $(66,67)$. The effect of hypo-fractionated RT on the immune response is investigated in both pre-clinical models and clinical trials. In HNSCC models, Morisada et al. irradiated mouse oral cancer cells with 2 and $8 \mathrm{~Gy}$, both in vitro and in vivo, and demonstrated dose-dependent antigen release, antigenspecific T-cells activation, and cytotoxic targeting of cells in both models. Importantly, the effect that followed 8 Gy was greater than the effect achieved after administering 2 Gy (68). The same group also combined PD-1 blockade with hypofractionated RT given in two doses; two fractions of 8 Gy and ten fractions of 2 Gy. The hypo-fractionated high dose preserved and even enhanced anti-tumor immunity, and when it was combined with PD-1 blockade, better control of primary and distant tumors was achieved (69). It has also been shown that PD-L1 upregulation following radiation can limit local control of tumors in murine models and that blockade of the PD-1/PD-L1 axis concurrently with radiation results in enhanced tumor control (70). These and other pre-clinical studies have provided a rationale for investigating the combination of RT and immunotherapy in human clinical trials.

\section{Combination of radiation therapy with immunotherapy}

Although immunotherapy with checkpoint inhibitors targeting PD-1 has been shown to improve OS in patients with R/M HNSCC, response rates to monotherapy are only $13-17 \%(37,41-43)$. To improve immunotherapy response rates, there has recently been a surge of interest in combining checkpoint inhibitors with radiation (59). The rationale for these studies is that the addition of radiation will incrementally improve the systemic response seen with PD-1 blockade via a radiation in situ vaccination effect that will propagate via epitope spread, or other means, to ultimately drive a systemic anti-tumor immune response beyond the locally irradiated field. To date, evidence of such effect in R/M HNSCC or other solid tumors is lacking, and a recently reported phase 2 randomized clinical trial failed to show any difference in response rate between patients treated with anti-PD-1 vs. those treated with anti-PD-1 and SBRT ( $\mathrm{n}=62$, objective response rate $34.5 \%$ vs. $29.0 \%$, 95\% CI: $19.9-52.7 \%, 16.1-46.6 \%$, respectively) (71). An alternative but an equally plausible hypothesis is that notwithstanding a weak in situ vaccine effect, the addition of PD-1 blockade to RT will modulate the TME and exert a far greater influence on the local response via blockade of upregulated PD-L1 in tumor following RT, leading to enhanced immune-mediated tumor killing. It has been shown that PD-L1 upregulation following radiation can limit local control of tumors in murine models and that blockade of the PD-1/PD-L1 axis concurrent with RT results in enhanced tumor control (70). The addition of avelumab (PD-1 inhibitor) to CRT in unresected locally advanced HNSCC was assessed in a randomized, doubleblind, placebo-controlled phase 3 clinical trial. Median PFS was not reached in the avelumab group ( $\mathrm{n}=350,95 \%$ CI, 16.9 months) or in the placebo group ( $\mathrm{n}=347,23.0$ months), with a stratified HR of 1.21 in favor of placebo (95\% CI, $0.93-1.57)$ (72). These data suggest that the addition of PD-1 inhibitor may lead to a different outcome in primary $v s$. locally advanced HNSCC and if combined with surgery vs. definitive CRT.

Ongoing trials aim to combine SBRT and PD-1 blockade in the adjuvant and neoadjuvant settings. Study NCT03247712 has completed the recruitment of 21 primary HNSCC patients to receive three doses of nivolumab and 3 or 5 fractions of 8 Gy prior to restaging and surgical resection. Following surgery, the patients received three additional doses of nivolumab. Outcome measures include an unplanned delay to surgery (safety and tolerability of neoadjuvant treatment), reduced tumor size, and reduction in lymph nodes size. The results of this study were recently published, demonstrating $86 \%$ MPR and $67 \%$ pCR (73). Study NCT03618134 by UCLA, Jonsson Comprehensive Cancer Center, is recruiting primary HPV- 
positive oropharyngeal SCC patients to receive durvalumab (with or without tremelimumab) and undergo SBRT. Between weeks 6-8, patients will undergo trans-oral robotic surgery and neck dissection, and on week 12 , they will be administered with durvalumab every four weeks up to 4 doses. Study NCT03635164 by the University of Colorado, Denver recruited primary HPV-negative HNSCC patients to receive one dose of neoadjuvant durvalumab approximately 3-6 weeks prior to standard-of-care surgery. It is given concurrently with the first dose of radiation, which includes two fractions of 6 Gy and, if possible, three fractions of 6 Gy. Patients receive up to 6 doses of durvalumab and undergo radiation postoperatively.

\section{Combination therapies-immunotherapy and chemotherapy}

A combined effect of chemotherapy and immunotherapy is evident while treating various kinds of cancer. Mapping the tumoral environment of breast cancer and colorectal cancer patients who responded to chemotherapy reveals many T-cells and a shift towards cytotoxic $\mathrm{CD}^{+}{ }^{+} \mathrm{T}$-cells over Treg cells $(74,75)$. The underlying mechanism is assumed to be chemotherapy-driven tumor cell death, which leads to the activation of T-cells and antigen presentation by DC. A fundamental concept in this process is defining tumor stress and tumor death as immunogenic (76). Several studies have demonstrated the crucial role of a competent immune response as a prerequisite for successful CRT. Among these are pre-clinical models in which chemotherapy was administered in parallel to immunogenic cell death induction but yielded optimal response only in immunocompetent mice (76-78). Clinical trials in NSCLC $(79,80)$ and triple-negative breast cancer (81) have resulted in encouraging results and are pointing to the interdependency of chemotherapy and immunotherapy and the therapeutic effect exerted by this synergy. In the phase 3 study NCT02358031, R/M HNSCC patients ( $\mathrm{n}=882)$ were randomly allocated into three groups: Pembrolizumab alone, pembrolizumab plus a platinum, and 5 -FU, or cetuximab plus a platinum and 5-FU (EXTREME). Benefit in OS was demonstrated for the combination of pembrolizumab plus a platinum and 5-FU over EXTREME for the entire population (13.0 vs. 10.7 months, HR 0.77, 95\% CI: $0.63-0.93, \mathrm{P}=0.0034)$, and for pembrolizumab alone over EXTREME for patients with a combined positive score of PD-L1 expression (CPS) $\geq 1$ (12.3 vs. 10.3 months, HR 0.78, 95\% CI: 0.64-0.96, $\mathrm{P}=0.0086)$ (41). This has led to the FDA approval for pembrolizumab alone as first-line therapy for patients with CPS $\geq 1$ and for pembrolizumab plus a platinum and $5-\mathrm{FU}$ for the entire population, regardless of CPS. Importantly, this study demonstrated the added value for each treatment modality: Pembrolizumab increased OS as long as CPS $\geq 1$, it has led to a lower rate of adverse events, and its response rate was more durable with a median duration of 22.6 months compared to 4.5 with EXTREME. However, the response rate to EXTREME was superior to that of pembrolizumab alone (36\% vs. $17 \%$, respectively), and the rate of progressive disease was better in EXTREME compared to pembrolizumab alone (12\% vs. $41 \%$, respectively) (41). These data demonstrate the diversity among patients and the substantial effect of the TME on the outcome of a given treatment. Accordingly, combining pembrolizumab and chemotherapy may be more appropriate for patients with lower CPS scores and a need for a rapid response rate, while pembrolizumab alone may be preferred for a less symptomatic state of disease and a high CPS score (32). Providing the appropriate combination for each patient and unveiling the factors that determine a successful outcome is essential in the studies that should follow.

\section{Intratumoral immunotherapy}

Over the years, therapeutic agents were introduced into tumors using variable techniques and substances to achieve several potential advantages. First, focusing the desired effect at the target area while limiting unwanted impact at other sites; second, using a reduced amount of medication for each patient while maintaining a high concentration at the tumor site; and third, maximizing the immune response by utilizing the direct interaction between the agent and the TME (82). Intratumoral and intertumoral heterogeneity pose a significant challenge for treatment, as it necessitates the targeting of different antigens throughout the tumor and across remote sites. Introducing an immune-stimulator directly into the tumor may overcome this major obstacle by utilizing the specific TME and the host's immune system, potentially negating the need for antigen isolation and exogenous treatment personalization (82). Over the years, several techniques were developed and tested for the delivery of various agents to induce an anti-tumoral immune response. The delivery methods include direct injection, image-guided injection, electroporation, and nano/microdelivery. The therapeutic agents are divided into bacteriaderived agents, oncolytic viruses, immune-modulators, cytokines, and chemotherapeutic agents. Methods that are 
relevant to HNSCC will be described next.

\section{Cytokines}

Since cytokines are vital components of the immune response, they form a natural target for cancer therapy. Interleukin 2 (IL-2) is synthesized by $\mathrm{CD}^{+}{ }^{+} \mathrm{T}$-cells, and it constitutes a key factor for the maintenance of Treg's and the differentiation of effector T-cells following antigen-mediated activation (83). Manipulation of IL-2 may stimulate an immune response by induction of $\mathrm{CD} 8^{+}$ T-cells or suppressing it by expanding Treg's (83). Systemic treatment with high-dose IL-2 (aldesleukin) to melanoma and renal cancer patients has evolved over the years, being integrated with adoptive cell transfer, non-myeloablative chemotherapy, and total body irradiation demonstrating a continually improving response rate (84). However, this treatment has been associated with severe toxicities to the heart, lungs, kidneys, and central nervous system (85). To minimize systemic adverse effects, IL-2 was injected intralesionally to 48 patients with advanced melanoma metastases; the results showed an impressive complete response rate of more than $60 \%$ while limiting toxicities to grade 1 and 2 (86). Several trials have focused on HNSCC patients undergoing perilymphatic injections of IL-2 with contradictory results. Earlier studies resulted in a temporary partial or complete response followed by a relapse in inoperable cases (87), a low ORR in advanced HNSCC (88), and no benefit in clinical outcome when combined with surgery and RT (89). In contrast, later studies demonstrated prolonged disease-free survival (DFS) and OS following neoadjuvant IL-2 perilymphatic injection prior to surgery and RT ( $\mathrm{n}=201,5$ years OS $73 \%, 55 \%$, DFS $64 \%, 51 \%$, IL-2 vs. control, respectively) (90). Studies also focus on a mixture of several cytokines, as in the case of IRX-2, a human donor-derived mix containing IL-1 $\beta$, IL-2, IL-6, IL-8, IFNg, TNFa, and GMCSF (91). Prolonged survival and delay in recurrence were demonstrated following neoadjuvant perilymphatic injections of IRX-2 before surgery and RT according to the standard of care (92). INSPIRE is an ongoing phase 2 study of neoadjuvant and adjuvant IRX-2 in patients with newly diagnosed stage IIIVa oral SCC (NCT02609386).

TNFerade Biologic is an adenoviral vector inserted with a human TNFa gene that is constructed to be induced by radiation. In a phase 1 escalation study conducted on 14 recurrent HNSCC patients, TNFerade Biologic was injected into the tumor concurrently with CRT. The study met its safety and dosage outcomes with a response rate of $83.3 \%$ and a median survival rate of 9.6 months (93).

Increased level of IL-12 is among the causes for differentiation of T-cells into Th1 cells, which consequently leads to $\mathrm{CD} 8^{+} \mathrm{T}$-cell-mediated adaptive immunity (17), and its level has been associated with improved clinical outcomes (18). Tavokinogene Telseplasmid is a DNA plasmid that encodes for $\mathrm{p} 35$ and p40 subunits of IL-12, and it is administered intratumorally followed by electroporation. This protocol was studied in a phase 1 trial conducted on 19 advanced melanoma patients, and results showed complete regression in two patients and partial response or disease stabilization in eight patients (94). Importantly, unlike the systemic administration of IL-12, the intratumoral administration was well tolerated, and additional clinical trials in melanoma patients are ongoing (95). Study NCT03823131 by UCSF Medical Center-Mount Zion, San Francisco, will recruit R/M HNSCC patients to receive intratumoral Tavokinogene Telseplasmid by electroporation, together with pembrolizumab and epacadostat (inhibitor of Indoleamine 2,3 dioxygenase, a negative regulator of immune responses) (96).

\section{STING}

STING (STimulator of INterferon Genes) is a critical component of the innate immune response in detecting certain viruses and intracellular DNA and induction of type I interferon production as part of cellular host defense $(97,98)$. To harness this mechanism to induce an anti-tumoral immune response, STING agonists were injected into an established murine B16 melanoma tumor, 4T-1 colon tumor, and CT26 mammary tumor, leading to a durable regression and a potent anti-tumor T-cell response that was mainly dependent upon $\mathrm{CD}^{+}$cells (99). Interestingly, the anti-tumoral effect included the rejection of non-injected tumors (abscopal effect), clearance of metastases, and successful immune response to autologous tumor rechallenge (99).

In the field of HNSCC, the murine immunogenic MOC1 and non-immunogenic MOC2 models were used to study the effect of intratumoral injection of cyclic dinucleotide $(\mathrm{CDN})$, a STING pathway activator. While the injection of CDN to MOC1 established tumors resulted in a complete regression rate of $50 \%$ and growth inhibition of the remaining tumors, only a slight delay in tumor growth was observed in the MOC2 group. Treating MOC1 in STING-deficient mice and $\mathrm{CD} 8^{+}$depleted mice eliminated 
the anti-tumor response, while the addition of systemic PD-L1 antibody treatment to the intratumoral injection increased the complete regression rate to $90 \%$ (100). This study underlines the critical role of $\mathrm{CD}^{+}$cells in the immune response to STING activation and the potential of incorporating intratumoral priming of immune response into existing checkpoint inhibition regimens.

Baird et al. performed subtotal tumor resection in several different murine HNSCC models, followed by intratumoral administration of STING ligand incorporated into Matrigel hydrogel. This treatment resulted in a cure of the residual tumor while all the control group members recurred (101). These data demonstrate that STING ligands act as potent local immunotherapy that controls residual tumors following surgical resection in HNSCC pre-clinical models and suggests a role for implantable immune-modulating interventions to improve loco-regional control in human patients.

\section{Future prospects}

\section{Vaccines}

Vaccine therapy aims at creating a durable immune response that will outlast the tumor cells and carcinogenesis. HPV is a principal target for vaccine therapy, and significant resources are being put into its continuing development and application. The causal effect of HPV on the development of HNSCC was described for the first time in 2000, after identifying its surrogate marker p16 in $25 \%$ of HNSCC specimens (102). It is known today that the combined expression of p16 (tumor suppressor gene) and HPV DNA in a specimen constitutes a favorable prognostic factor of HNSCC (14). Evaluation of the TME reveals a higher level of TIL in HPV-positive than in HPV-negative HNSCC specimens, indicating a more robust activation of the antitumoral immune response in $\mathrm{HPV}$-positive HNSCC $(103,104)$. Moreover, HPV-positive HNSCC tumors are more radiosensitive as compared to $\mathrm{HPV}$-negative tumors (105). These data underline the importance of designing different approaches for treating HNSCC patients based on their HPV status.

Of particular interest is the potential link between the HPV vaccine and oropharyngeal cancer. HPV vaccines were reported in phase 3 trials as preventing persistent genital lesions and premalignant genital lesions (106). The causal effect of the HPV vaccine and oropharyngeal cancer has not been identified to date, but a few trials have already begun to study this possible link. Phase 1 dose-escalation trial concentrated on HPV16 and MAGE-A3 vaccines given in four doses subcutaneously to R/M HNSCC patients who are positive for HPV16 and MAGE-A3, respectively. T-cell and antibody responses were observed, and the vaccines were reported to be well tolerated (107).

DC-based vaccines are mainly used in other types of cancer and, to a lesser degree, also in HNSCC. A phase 1 clinical trial focused on $\mathrm{p} 53$ as a target for a DC-based vaccine delivered into inguinal lymph nodes of sixteen HNSCC patients at three different time points. Results showed an elevated frequency of p53-specific T-cells, improved phenotype and function of DC, and a favorable two-year survival rate of $88 \%$ (108). In another study, autologous DC loaded with apoptotic tumor cells were used to formulate a vaccine from HNSCC resected tumors. Among the thirty patients initially enrolled in this study, only four were eventually administered with the vaccine due to a small number of cases in which the pre-vaccination criteria were met. Although all four patients benefited from the vaccine, the authors concluded that this vaccine type would only be relevant for a small number of patients that exhibit delayed-type hypersensitivity and only if a sufficient amount of sterile tumor cells has been produced (109).

The quantity of studies in which neoadjuvant vaccines are administered to HNSCC patients remains small. As studies ensue, the benefit from vaccinating the general population and HNSCC patients against HPV remains to be seen.

\section{Microparticles and nanoparticles}

While surgery is considered the standard of care for most solid tumors, it does not necessarily eliminate the tumor in its entirety; thus, effective adjuvant therapy to counteract residual disease is required. Novel drug delivery technologies are constantly evolving, and significant progress is seen in post-surgical adjuvant treatment. Translation of these technologies to the clinical setting can transform the field of surgical oncology by reducing recurrence rates and metastasis, shifting treatment paradigms, and improving survival rates (110). To deliver immunotherapeutic agents directly into the TME over a more extended period, PD-1 and CTLA-4 inhibitors were encapsulated in dextran nanoparticles integrated with hyaluronic acid and introduced into a transdermal microneedle patch that was applied onto melanomabearing mice. Based on a preliminary in vitro assay that 
showed a sustained release period of three days, the authors showed a benefit in survival and a synergistic effect of this combination (111). The same group has also succeeded in conjugating PD-1 inhibitor onto platelets for its delivery into the TME, utilizing the natural migration of platelets to the tumor site. Following resection of the majority of melanoma and triple-negative mammary carcinoma, intentionally leaving behind residual tumor, mice were treated with intravenous platelets conjugated with a PD-1 inhibitor. The circulating half-life of this construct proved to be over six times longer than the unconjugated PD-1 inhibitor, and it has led to an increased amount of TIL (112). These novel delivery methods demonstrate the potential of local delivery of immunomodulating agents using sustainedrelease methods that prime the immune response locally and systemically.

\section{Adoptive T-cell therapy}

To harness the potential of specific cell populations to target a patient's tumor, two adoptive T-cell therapy (ACT) methods were developed: (I) TIL are isolated from surgically resected specimens; (II) T-cells are derived from the peripheral blood and genetically modified ex vivo to target tumor cells. In both techniques, cells are expanded ex vivo and reinfused to the patient (113). Initially tested in metastatic melanoma in 1988 (114), ACT was further developed and produced objective clinical response rates of $42-51 \%$ among refractory metastatic melanoma patients (115-118). Utilizing a similar methodology, TIL were successfully expanded from HNSCC specimens by Junker et al. in 2011 (119), later evolving into clinical trials of ACT in HNSCC. Studies NCT03083873 and NCT03645928 by Iovance Biotherapeutics, Inc. are currently evaluating TIL infusion (LN-145/LN-145-S1) to R/M HNSCC patients.

\section{Conclusions}

Cancer immunotherapy is a validated and transformational approach for treating patients with HNSCC. However, predictably modulating the immune response to overcome existing cancer and its future spread poses a significant challenge in pre-clinical models and patients. Despite the successful application of immunotherapy across multiple tumor types, durable response and cure remain elusive. Successful immunologic elimination of cancer is complex and challenged by a series of biological steps to dampen the immune response, with multiple negative feedback loops and checkpoints that enable control of anti-tumor immunity. Adding to this complexity is that the cancer itself is heterogeneous and the product of genetic mutations can, on one hand, lead to cancer progression, but on the other hand can serve as an important antigenic target of immune response. Large scale efforts are underway to test novel combinations of immunotherapy with other immunotherapy agents, targeted small molecules, chemotherapy, RT and surgery. Future success will be dependent upon better understanding of the synergies between therapies and the effect on anti-cancer immune response.

\section{Acknowledgments}

Funding: This work was supported by grants from the Oral and Maxillofacial Surgery Foundation and NIH (No. 1R01CA234343-01A1).

\section{Footnote}

Provenance and Peer Review: This article was commissioned by the Guest Editor (Sung-Kiang Chuang) for the series "Clinical Outcomes and Innovations in Oral and Maxillofacial Surgery" published in Frontiers of Oral and Maxillofacial Medicine. The article has undergone external peer review.

Reporting Checklist: The authors have completed the Narrative Review reporting checklist. Available at https://fomm. amegroups.com/article/view/10.21037/fomm-21-48/rc

Conflicts of Interest: Both authors have completed the ICMJE uniform disclosure form (available at https://fomm. amegroups.com/article/view/10.21037/fomm-21-48/ coif). The series "Clinical Outcomes and Innovations in Oral and Maxillofacial Surgery" was commissioned by the editorial office without any funding or sponsorship. RBB reports that this work was supported by grants from the Oral and Maxillofacial Surgery Foundation and NIH (No. 1R01CA234343-01A1). RBB serves as an unpaid editorial board member of Frontiers of Oral and Maxillofacial Medicine from August 2021 to July 2023. RBB receives research funding from BMS and Merck, payment from the Speakers bureau of Regeneron and Merck, and is in the advisory board of MacroGenics. The authors have no other conflicts of interest to declare.

Ethical Statement: The authors are accountable for all 
aspects of the work in ensuring that questions related to the accuracy or integrity of any part of the work are appropriately investigated and resolved.

Open Access Statement: This is an Open Access article distributed in accordance with the Creative Commons Attribution-NonCommercial-NoDerivs 4.0 International License (CC BY-NC-ND 4.0), which permits the noncommercial replication and distribution of the article with the strict proviso that no changes or edits are made and the original work is properly cited (including links to both the formal publication through the relevant DOI and the license). See: https://creativecommons.org/ licenses/by-nc-nd/4.0/.

\section{References}

1. Ferlay J, Soerjomataram I, Dikshit R, et al. Cancer incidence and mortality worldwide: sources, methods and major patterns in GLOBOCAN 2012. Int J Cancer 2015;136:E359-86.

2. Cancer facts and figures 2021 - American Cancer Society 2021. Available online: https://www.cancer.org/research/ cancer-facts-statistics/all-cancer-facts-figures/cancer-factsfigures-2021.html

3. Berman TA, Schiller JT. Human papillomavirus in cervical cancer and oropharyngeal cancer: One cause, two diseases. Cancer 2017;123:2219-29.

4. O'Rorke MA, Ellison MV, Murray LJ, et al. Human papillomavirus related head and neck cancer survival: a systematic review and meta-analysis. Oral Oncol 2012;48:1191-201.

5. Langendijk JA, Doornaert P, Verdonck-de Leeuw IM, et al. Impact of late treatment-related toxicity on quality of life among patients with head and neck cancer treated with radiotherapy. J Clin Oncol 2008;26:3770-6.

6. Weinstein GS, O'Malley BW Jr, Magnuson JS, et al. Transoral robotic surgery: a multicenter study to assess feasibility, safety, and surgical margins. Laryngoscope 2012;122:1701-7.

7. Cramer JD, Hicks KE, Rademaker AW, et al. Validation of the eighth edition American Joint Committee on Cancer staging system for human papillomavirus-associated oropharyngeal cancer. Head Neck 2018;40:457-66.

8. Quon H, Forastiere AA. Controversies in treatment deintensification of human papillomavirus-associated oropharyngeal carcinomas: should we, how should we, and for whom? J Clin Oncol 2013;31:520-2.
9. Yom SS, Torres-Saavedra P, Caudell JJ, et al. NRGHN002: A Randomized Phase II Trial for Patients With p16-Positive, Non-Smoking-Associated, Locoregionally Advanced Oropharyngeal Cancer. Int J Radiat Oncol 2019;105:684-5.

10. Albergotti WG, Jordan J, Anthony K, et al. A prospective evaluation of short-term dysphagia after transoral robotic surgery for squamous cell carcinoma of the oropharynx. Cancer 2017;123:3132-40.

11. Sharma A, Patel S, Baik FM, et al. Survival and Gastrostomy Prevalence in Patients With Oropharyngeal Cancer Treated With Transoral Robotic Surgery vs Chemoradiotherapy. JAMA Otolaryngol Head Neck Surg 2016;142:691-7.

12. Nichols AC, Theurer J, Prisman E, et al. Radiotherapy versus transoral robotic surgery and neck dissection for oropharyngeal squamous cell carcinoma (ORATOR): an open-label, phase 2, randomised trial. Lancet Oncol 2019;20:1349-59.

13. León X, Hitt R, Constenla M, et al. A retrospective analysis of the outcome of patients with recurrent and/or metastatic squamous cell carcinoma of the head and neck refractory to a platinum-based chemotherapy. Clin Oncol (R Coll Radiol) 2005;17:418-24.

14. Chaudhary S, Ganguly K, Muniyan S, et al. Immunometabolic Alterations by HPV Infection: New Dimensions to Head and Neck Cancer Disparity. J Natl Cancer Inst 2019;111:233-44.

15. Shitara K, Nishikawa H. Regulatory T cells: a potential target in cancer immunotherapy. Ann N Y Acad Sci 2018;1417:104-15.

16. Chen $\mathrm{X}, \mathrm{Du} \mathrm{Y}, \mathrm{Lin} \mathrm{X}$, et al. CD4+CD25+ regulatory $T$ cells in tumor immunity. Int Immunopharmacol 2016;34:244-9.

17. Szabo SJ, Sullivan BM, Peng SL, et al. Molecular mechanisms regulating Th1 immune responses. Annu Rev Immunol 2003;21:713-58.

18. Galon J, Costes A, Sanchez-Cabo F, et al. Type, density, and location of immune cells within human colorectal tumors predict clinical outcome. Science 2006;313:1960-4.

19. Strauss L, Bergmann C, Whiteside TL. Functional and phenotypic characteristics of CD4+CD25highFoxp3+ Treg clones obtained from peripheral blood of patients with cancer. Int J Cancer 2007;121:2473-83.

20. Gaur P, Singh AK, Shukla NK, et al. Inter-relation of Th1, Th2, Th17 and Treg cytokines in oral cancer patients and their clinical significance. Hum Immunol 
2014;75:330-7.

21. Maggioni D, Pignataro L, Garavello W. T-helper and T-regulatory cells modulation in head and neck squamous cell carcinoma. Oncoimmunology 2017;6:e1325066.

22. Hansen M, Andersen MH. The role of dendritic cells in cancer. Semin Immunopathol 2017;39:307-16.

23. Tang M, Diao J, Cattral MS. Molecular mechanisms involved in dendritic cell dysfunction in cancer. Cell Mol Life Sci 2017;74:761-76.

24. Steinman RM, Hawiger D, Nussenzweig MC. Tolerogenic dendritic cells. Annu Rev Immunol 2003;21:685-711.

25. Broz ML, Binnewies M, Boldajipour B, et al. Dissecting the tumor myeloid compartment reveals rare activating antigen-presenting cells critical for $\mathrm{T}$ cell immunity. Cancer Cell 2014;26:638-52.

26. Krall JA, Reinhardt F, Mercury OA, et al. The systemic response to surgery triggers the outgrowth of distant immune-controlled tumors in mouse models of dormancy. Sci Transl Med 2018;10:eaan3464.

27. Dillekås H, Demicheli R, Ardoino I, et al. The recurrence pattern following delayed breast reconstruction after mastectomy for breast cancer suggests a systemic effect of surgery on occult dormant micrometastases. Breast Cancer Res Treat 2016;158:169-78.

28. Freeman GJ, Long AJ, Iwai Y, et al. Engagement of the PD-1 immunoinhibitory receptor by a novel B7 family member leads to negative regulation of lymphocyte activation. J Exp Med 2000;192:1027-34.

29. Yang WF, Wong MCM, Thomson PJ, et al. The prognostic role of PD-L1 expression for survival in head and neck squamous cell carcinoma: A systematic review and meta-analysis. Oral Oncol 2018;86:81-90.

30. Ferris RL. Immunology and Immunotherapy of Head and Neck Cancer. J Clin Oncol 2015;33:3293-304.

31. Rischin D, Harrington KJ, Greil R, et al. Protocolspecified final analysis of the phase 3 KEYNOTE-048 trial of pembrolizumab (pembro) as first-line therapy for recurrent/metastatic head and neck squamous cell carcinoma (R/M HNSCC). J Clin Oncol 2019;37:6000.

32. Cramer JD, Burtness B, Ferris RL. Immunotherapy for head and neck cancer: Recent advances and future directions. Oral Oncol 2019;99:104460.

33. Bonner JA, Harari PM, Giralt J, et al. Radiotherapy plus cetuximab for squamous-cell carcinoma of the head and neck. N Engl J Med 2006;354:567-78.

34. Bonner JA, Harari PM, Giralt J, et al. Radiotherapy plus cetuximab for locoregionally advanced head and neck cancer: 5-year survival data from a phase 3 randomised trial, and relation between cetuximab-induced rash and survival. Lancet Oncol 2010;11:21-8.

35. Gillison ML, Trotti AM, Harris J, et al. Radiotherapy plus cetuximab or cisplatin in human papillomavirus-positive oropharyngeal cancer (NRG Oncology RTOG 1016): a randomised, multicentre, non-inferiority trial. Lancet 2019;393:40-50.

36. Mehanna H, Robinson M, Hartley A, et al. Radiotherapy plus cisplatin or cetuximab in low-risk human papillomavirus-positive oropharyngeal cancer (DeESCALaTE HPV): an open-label randomised controlled phase 3 trial. Lancet 2019;393:51-60.

37. Ferris RL, Blumenschein G Jr, Fayette J, et al. Nivolumab for Recurrent Squamous-Cell Carcinoma of the Head and Neck. N Engl J Med 2016;375:1856-67.

38. Ferris RL, Blumenschein G Jr, Fayette J, et al. Nivolumab vs investigator's choice in recurrent or metastatic squamous cell carcinoma of the head and neck: 2-year long-term survival update of CheckMate 141 with analyses by tumor PD-L1 expression. Oral Oncol 2018;81:45-51.

39. Even C, Daste A, Saada-Bouzid E, et al. A safety study of nivolumab in patients with recurrent and/or metastatic platinum-refractory squamous cell carcinoma of the head and neck (R/M SCCHN): Interim analysis on 199 patients - The TOPNIVO study on behalf of the GORTEC and the Unicancer Head \& Neck Group. J Clin Oncol 2019;37:6032.

40. Cohen EEW, Soulières D, Le Tourneau C, et al. Pembrolizumab versus methotrexate, docetaxel, or cetuximab for recurrent or metastatic head-and-neck squamous cell carcinoma (KEYNOTE-040): a randomised, open-label, phase 3 study. Lancet 2019;393:156-67.

41. Burtness B, Harrington KJ, Greil R, et al. Pembrolizumab alone or with chemotherapy versus cetuximab with chemotherapy for recurrent or metastatic squamous cell carcinoma of the head and neck (KEYNOTE-048): a randomised, open-label, phase 3 study. Lancet 2019;394:1915-28.

42. Seiwert TY, Burtness B, Mehra R, et al. Safety and clinical activity of pembrolizumab for treatment of recurrent or metastatic squamous cell carcinoma of the head and neck (KEYNOTE-012): an open-label, multicentre, phase $1 \mathrm{~b}$ trial. Lancet Oncol 2016;17:956-65.

43. Chow LQM, Haddad R, Gupta S, et al. Antitumor Activity of Pembrolizumab in Biomarker-Unselected Patients With Recurrent and/or Metastatic Head and Neck Squamous Cell Carcinoma: Results From the Phase 
Ib KEYNOTE-012 Expansion Cohort. J Clin Oncol 2016;34:3838-45.

44. Bauml J, Seiwert TY, Pfister DG, et al. Pembrolizumab for Platinum- and Cetuximab-Refractory Head and Neck Cancer: Results From a Single-Arm, Phase II Study. J Clin Oncol 2017;35:1542-9.

45. Harrington KJ, Soulières D, Le Tourneau C, et al. Quality of Life With Pembrolizumab for Recurrent and/ or Metastatic Head and Neck Squamous Cell Carcinoma: KEYNOTE-040. J Natl Cancer Inst 2021;113:171-81.

46. Forde PM, Chaft JE, Pardoll DM. Neoadjuvant PD-1 Blockade in Resectable Lung Cancer. N Engl J Med 2018;379:e14.

47. Huang AC, Orlowski RJ, Xu X, et al. A single dose of neoadjuvant PD-1 blockade predicts clinical outcomes in resectable melanoma. Nat Med 2019;25:454-61.

48. Cottrell TR, Thompson ED, Forde PM, et al. Pathologic features of response to neoadjuvant anti-PD-1 in resected non-small-cell lung carcinoma: a proposal for quantitative immune-related pathologic response criteria (irPRC). Ann Oncol 2018;29:1853-60.

49. Uppaluri R, Campbell KM, Egloff AM, et al. Neoadjuvant and Adjuvant Pembrolizumab in Resectable Locally Advanced, Human Papillomavirus-Unrelated Head and Neck Cancer: A Multicenter, Phase II Trial. Clin Cancer Res 2020;26:5140-52.

50. Schoenfeld JD, Hanna GJ, Jo VY, et al. Neoadjuvant Nivolumab or Nivolumab Plus Ipilimumab in Untreated Oral Cavity Squamous Cell Carcinoma: A Phase 2 Open-Label Randomized Clinical Trial. JAMA Oncol 2020;6:1563-70.

51. Wara WM. Immunosuppression associated with radiation therapy. Int J Radiat Oncol Biol Phys 1977;2:593-6.

52. Walle T, Martinez Monge R, Cerwenka A, et al. Radiation effects on antitumor immune responses: current perspectives and challenges. Ther Adv Med Oncol 2018;10:1758834017742575.

53. Sharabi AB, Lim M, DeWeese TL, et al. Radiation and checkpoint blockade immunotherapy: radiosensitisation and potential mechanisms of synergy. Lancet Oncol 2015;16:e498-509.

54. Postow MA, Callahan MK, Barker CA, et al. Immunologic correlates of the abscopal effect in a patient with melanoma. N Engl J Med 2012;366:925-31.

55. Perez CA, Fu A, Onishko H, et al. Radiation induces an antitumour immune response to mouse melanoma. Int $\mathrm{J}$ Radiat Biol 2009;85:1126-36.

56. Golden EB, Frances D, Pellicciotta I, et al. Radiation fosters dose-dependent and chemotherapyinduced immunogenic cell death. Oncoimmunology 2014;3:e28518.

57. Rodriguez-Ruiz ME, Garasa S, Rodriguez I, et al. Intercellular Adhesion Molecule-1 and Vascular Cell Adhesion Molecule Are Induced by Ionizing Radiation on Lymphatic Endothelium. Int J Radiat Oncol Biol Phys 2017;97:389-400.

58. Kachikwu EL, Iwamoto KS, Liao YP, et al. Radiation enhances regulatory $\mathrm{T}$ cell representation. Int $\mathrm{J}$ Radiat Oncol Biol Phys 2011;81:1128-35.

59. Bernstein MB, Krishnan S, Hodge JW, et al. Immunotherapy and stereotactic ablative radiotherapy (ISABR): a curative approach? Nat Rev Clin Oncol 2016;13:516-24.

60. Park C, Papiez L, Zhang S, et al. Universal survival curve and single fraction equivalent dose: useful tools in understanding potency of ablative radiotherapy. Int J Radiat Oncol Biol Phys 2008;70:847-52.

61. Potters L, Kavanagh B, Galvin JM, et al. American Society for Therapeutic Radiology and Oncology (ASTRO) and American College of Radiology (ACR) practice guideline for the performance of stereotactic body radiation therapy. Int J Radiat Oncol Biol Phys 2010;76:326-32.

62. Khan L, Tjong M, Raziee H, et al. Role of stereotactic body radiotherapy for symptom control in head and neck cancer patients. Support Care Cancer 2015;23:1099-103.

63. Siddiqui F, Patel M, Khan M, et al. Stereotactic body radiation therapy for primary, recurrent, and metastatic tumors in the head-and-neck region. Int J Radiat Oncol Biol Phys 2009;74:1047-53.

64. Hellman S, Weichselbaum RR. Oligometastases. J Clin Oncol 1995;13:8-10.

65. Pastorino U, Buyse M, Friedel G, et al. Long-term results of lung metastasectomy: prognostic analyses based on 5206 cases. J Thorac Cardiovasc Surg 1997;113:37-49.

66. Formenti SC, Demaria S. Radiation therapy to convert the tumor into an in situ vaccine. Int J Radiat Oncol Biol Phys 2012;84:879-80.

67. Demaria $\mathrm{S}, \mathrm{Ng} \mathrm{B}$, Devitt $\mathrm{ML}$, et al. Ionizing radiation inhibition of distant untreated tumors (abscopal effect) is immune mediated. Int J Radiat Oncol Biol Phys 2004;58:862-70.

68. Morisada M, Moore EC, Hodge R, et al. Dose-dependent enhancement of T-lymphocyte priming and CTL lysis following ionizing radiation in an engineered model of 
oral cancer. Oral Oncol 2017;71:87-94.

69. Morisada M, Clavijo PE, Moore E, et al. PD-1 blockade reverses adaptive immune resistance induced by highdose hypofractionated but not low-dose daily fractionated radiation. Oncoimmunology 2018;7:e1395996.

70. Gough MJ, Crittenden MR, Sarff M, et al. Adjuvant therapy with agonistic antibodies to CD134 (OX40) increases local control after surgical or radiation therapy of cancer in mice. J Immunother 2010;33:798-809.

71. McBride S, Sherman E, Tsai CJ, et al. Randomized Phase II Trial of Nivolumab With Stereotactic Body Radiotherapy Versus Nivolumab Alone in Metastatic Head and Neck Squamous Cell Carcinoma. J Clin Oncol 2021;39:30-7.

72. Lee NY, Ferris RL, Psyrri A, et al. Avelumab plus standardof-care chemoradiotherapy versus chemoradiotherapy alone in patients with locally advanced squamous cell carcinoma of the head and neck: a randomised, doubleblind, placebo-controlled, multicentre, phase 3 trial. Lancet Oncol 2021;22:450-62.

73. Leidner R, Crittenden M, Young K, et al. Neoadjuvant immunoradiotherapy results in high rate of complete pathological response and clinical to pathological downstaging in locally advanced head and neck squamous cell carcinoma. J Immunother Cancer 2021;9:e002485.

74. Halama N, Michel S, Kloor M, et al. Localization and density of immune cells in the invasive margin of human colorectal cancer liver metastases are prognostic for response to chemotherapy. Cancer Res 2011;71:5670-7.

75. Ladoire S, Mignot G, Dabakuyo S, et al. In situ immune response after neoadjuvant chemotherapy for breast cancer predicts survival. J Pathol 2011;224:389-400.

76. Kroemer G, Galluzzi L, Kepp O, et al. Immunogenic cell death in cancer therapy. Annu Rev Immunol 2013;31:51-72.

77. Apetoh L, Ghiringhelli F, Tesniere A, et al. Toll-like receptor 4-dependent contribution of the immune system to anticancer chemotherapy and radiotherapy. Nat Med 2007;13:1050-9.

78. Michaud M, Martins I, Sukkurwala AQ, et al. Autophagy-dependent anticancer immune responses induced by chemotherapeutic agents in mice. Science 2011;334:1573-7.

79. Gandhi L, Rodríguez-Abreu D, Gadgeel S, et al. Pembrolizumab plus Chemotherapy in Metastatic NonSmall-Cell Lung Cancer. N Engl J Med 2018;378:2078-92.

80. Horn L, Mansfield AS, Szczęsna A, et al. First-Line
Atezolizumab plus Chemotherapy in ExtensiveStage Small-Cell Lung Cancer. N Engl J Med 2018;379:2220-9.

81. Schmid P, Adams S, Rugo HS, et al. Atezolizumab and Nab-Paclitaxel in Advanced Triple-Negative Breast Cancer. N Engl J Med 2018;379:2108-21.

82. Marabelle A, Tselikas L, de Baere T, et al. Intratumoral immunotherapy: using the tumor as the remedy. Ann Oncol 2017;28:xii33-43.

83. Boyman O, Sprent J. The role of interleukin-2 during homeostasis and activation of the immune system. Nat Rev Immunol 2012;12:180-90.

84. Rosenberg SA. IL-2: the first effective immunotherapy for human cancer. J Immunol 2014;192:5451-8.

85. Schwartz RN, Stover L, Dutcher JP. Managing toxicities of high-dose interleukin-2. Oncology (Williston Park) 2002;16:11-20.

86. Weide B, Derhovanessian E, Pflugfelder A, et al. High response rate after intratumoral treatment with interleukin-2: results from a phase 2 study in 51 patients with metastasized melanoma. Cancer 2010;116:4139-46.

87. Cortesina G, De Stefani A, Galeazzi E, et al. Interleukin-2 injected around tumor-draining lymph nodes in head and neck cancer. Head Neck 1991;13:125-31.

88. Vlock DR, Snyderman CH, Johnson JT, et al. Phase Ib trial of the effect of peritumoral and intranodal injections of interleukin-2 in patients with advanced squamous cell carcinoma of the head and neck: an Eastern Cooperative Oncology Group trial. J Immunother Emphasis Tumor Immunol 1994;15:134-9.

89. De Stefani A, Valente G, Forni G, et al. Treatment of oral cavity and oropharynx squamous cell carcinoma with perilymphatic interleukin-2: clinical and pathologic correlations. J Immunother Emphasis Tumor Immunol 1996;19:125-33.

90. De Stefani A, Forni G, Ragona R, et al. Improved survival with perilymphatic interleukin 2 in patients with resectable squamous cell carcinoma of the oral cavity and oropharynx. Cancer 2002;95:90-7.

91. Wolf GT, Moyer JS, Kaplan MJ, et al. IRX-2 natural cytokine biologic for immunotherapy in patients with head and neck cancers. Onco Targets Ther 2018;11:3731-46.

92. Hadden J, Verastegui E, Barrera JL, et al. A trial of IRX-2 in patients with squamous cell carcinomas of the head and neck. Int Immunopharmacol 2003;3:1073-81.

93. Seiwert TY, Darga T, Haraf D, et al. A phase I dose 
escalation study of Ad GV.EGR.TNF.11D (TNFerade ${ }^{\mathrm{TM}}$ Biologic) with concurrent chemoradiotherapy in patients with recurrent head and neck cancer undergoing reirradiation. Ann Oncol 2013;24:769-76.

94. Daud AI, DeConti RC, Andrews S, et al. Phase I trial of interleukin-12 plasmid electroporation in patients with metastatic melanoma. J Clin Oncol 2008;26:5896-903.

95. Canton DA, Shirley S, Wright J, et al. Melanoma treatment with intratumoral electroporation of tavokinogene telseplasmid (pIL-12, tavokinogene telseplasmid). Immunotherapy 2017;9:1309-21.

96. Munn DH, Mellor AL. IDO in the Tumor Microenvironment: Inflammation, Counter-Regulation, and Tolerance. Trends Immunol 2016;37:193-207.

97. Ishikawa H, Barber GN. STING is an endoplasmic reticulum adaptor that facilitates innate immune signalling. Nature 2008;455:674-8.

98. Ishikawa H, Ma Z, Barber GN. STING regulates intracellular DNA-mediated, type I interferon-dependent innate immunity. Nature 2009;461:788-92.

99. Corrales L, Glickman LH, McWhirter SM, et al. Direct Activation of STING in the Tumor Microenvironment Leads to Potent and Systemic Tumor Regression and Immunity. Cell Rep 2015;11:1018-30.

100. Moore E, Clavijo PE, Davis R, et al. Established T CellInflamed Tumors Rejected after Adaptive Resistance Was Reversed by Combination STING Activation and PD-1 Pathway Blockade. Cancer Immunol Res 2016;4:1061-71.

101. Baird JR, Bell RB, Troesch V, et al. Evaluation of Explant Responses to STING Ligands: Personalized Immunosurgical Therapy for Head and Neck Squamous Cell Carcinoma. Cancer Res 2018;78:6308-19.

102. Gillison ML, Koch WM, Capone RB, et al. Evidence for a causal association between human papillomavirus and a subset of head and neck cancers. J Natl Cancer Inst 2000;92:709-20.

103. Russell S, Angell T, Lechner M, et al. Immune cell infiltration patterns and survival in head and neck squamous cell carcinoma. Head Neck Oncol 2013;5:24.

104.Krupar R, Robold K, Gaag D, et al. Immunologic and metabolic characteristics of HPV-negative and HPVpositive head and neck squamous cell carcinomas are strikingly different. Virchows Arch 2014;465:299-312.

105. Sethi S, Ali-Fehmi R, Franceschi S, et al. Characteristics and survival of head and neck cancer by HPV status: a cancer registry-based study. Int J Cancer 2012;131:1179-86. 106. Schiller JT, Castellsagué X, Garland SM. A review of clinical trials of human papillomavirus prophylactic vaccines. Vaccine 2012;30 Suppl 5:F123-38.

107.Zandberg DP, Rollins S, Goloubeva O, et al. A phase I dose escalation trial of MAGE-A3- and HPV16-specific peptide immunomodulatory vaccines in patients with recurrent/metastatic (RM) squamous cell carcinoma of the head and neck (SCCHN). Cancer Immunol Immunother 2015;64:367-79.

108. Schuler PJ, Harasymczuk M, Visus C, et al. Phase I dendritic cell $\mathrm{p} 53$ peptide vaccine for head and neck cancer. Clin Cancer Res 2014;20:2433-44.

109. Whiteside TL, Ferris RL, Szczepanski M, et al. Dendritic cell-based autologous tumor vaccines for head and neck squamous cell carcinoma. Head Neck 2016;38 Suppl 1:E494-501.

110. Bu LL, Yan J, Wang Z, et al. Advances in drug delivery for post-surgical cancer treatment. Biomaterials 2019;219:119182.

111. Wang C, Ye Y, Gu Z. Local delivery of checkpoints antibodies. Hum Vaccin Immunother 2017;13:245-8.

112. Wang C, Sun W, Ye Y, et al. In situ activation of platelets with checkpoint inhibitors for post-surgical cancer immunotherapy. Nat Biomed Eng 2017. doi: 10.1038/ s41551-016-0011.

113. Met Ö, Jensen KM, Chamberlain CA, et al. Principles of adoptive $\mathrm{T}$ cell therapy in cancer. Semin Immunopathol 2019;41:49-58.

114. Rosenberg SA, Packard BS, Aebersold PM, et al. Use of tumor-infiltrating lymphocytes and interleukin-2 in the immunotherapy of patients with metastatic melanoma. A preliminary report. N Engl J Med 1988;319:1676-80.

115.Dudley ME, Wunderlich JR, Yang JC, et al. Adoptive cell transfer therapy following non-myeloablative but lymphodepleting chemotherapy for the treatment of patients with refractory metastatic melanoma. J Clin Oncol 2005;23:2346-57.

116.Itzhaki O, Hovav E, Ziporen Y, et al. Establishment and large-scale expansion of minimally cultured "young" tumor infiltrating lymphocytes for adoptive transfer therapy. J Immunother 2011;34:212-20.

117. Radvanyi LG, Bernatchez C, Zhang M, et al. Specific lymphocyte subsets predict response to adoptive cell therapy using expanded autologous tumor-infiltrating lymphocytes in metastatic melanoma patients. Clin Cancer Res 2012;18:6758-70.

118. Andersen R, Donia M, Ellebaek E, et al. Long-Lasting Complete Responses in Patients with Metastatic Melanoma after Adoptive Cell Therapy with Tumor- 
Infiltrating Lymphocytes and an Attenuated IL2 Regimen. Clin Cancer Res 2016;22:3734-45.

119.Junker N, Andersen MH, Wenandy L, et al. Bimodal ex

doi: $10.21037 /$ fomm-21-48

Cite this article as: Sharon S, Bell RB. Immunotherapy in head and neck squamous cell carcinoma: a narrative review. Front Oral Maxillofac Med 2022;4:28. vivo expansion of $T$ cells from patients with head and neck squamous cell carcinoma: a prerequisite for adoptive cell transfer. Cytotherapy 2011;13:822-34. 